\title{
Undergraduate Texts in Mathematics
}

Editors

S. Axler

F.W. Gehring

K.A. Ribet

Springer Science+Business Media, LLC 
Abbott: Understanding Analysis.

Anglin: Mathematics: A Concise History and Philosophy.

Readings in Mathematics.

Anglin/Lambek: The Heritage of Thales.

Readings in Mathematics.

Apostol: Introduction to Analytic Number Theory. Second edition.

Armstrong: Basic Topology.

Armstrong: Groups and Symmetry.

Axler: Linear Algebra Done Right. Second edition

Beardon: Limits: A New Approach to Real Analysis.

Bak/Newman: Complex Analysis. Second edition.

Banchoff/Wermer: Linear Algebra Through Geometry. Second edition.

Berberian: A First Course in Real Analysis.

Bix: Conics and Cubics: $\mathrm{A}$ Concrete Introduction to Algebraic Curves.

Brémaud: An Introduction to Probabilistic Modeling.

Bressoud: Factorization and Primality Testing.

Bressoud: Second Year Calculus. Readings in Mathematics.

Brickman: Mathematical Introduction to Linear Programming and Game Theory.

Browder: Mathematical Analysis: An Introduction.

Buchmann: Introduction to Cryptography, Second edition.

Buskes/van Rooij: Topological Spaces: From Distance to Neighborhood.

Callahan: The Geometry of Spacetime: An Introduction to Special and General Relavitity.

Carter/van Brunt: The LebesgueStieltjes Integral: A Practical Introduction.

Cederberg: A Course in Modern Geometries. Second edition.
Childs: A Concrete Introduction to Higher Algebra. Second edition.

Chung/AitSahlia: Elementary Probability Theory: With Stochastic Processes and an Introduction to Mathematical Finance. Fourth edition.

Cox/Little/O'Shea: Ideals, Varieties, and Algorithms. Second edition.

Croom: Basic Concepts of Algebraic Topology.

Curtis: Linear Algebra: An Introductory Approach. Fourth edition.

Daepp/Gorkin: Reading, Writing, and Proving: A Closer Look at Mathematics.

Devlin: The Joy of Sets: Fundamentals of Contemporary Set Theory. Second edition.

Dixmier: General Topology.

Driver: Why Math?

Ebbinghaus/Flum/Thomas: Mathematical Logic. Second edition.

Edgar: Measure, Topology, and Fractal Geometry.

Elaydi: An Introduction to Difference Equations. Second edition.

Erdós/Surányi: Topics in the Theory of Numbers.

Estep: Practical Analysis in One Variable.

Exner: An Accompaniment to Higher Mathematics.

Exner: Inside Calculus.

Fine/Rosenberger: The Fundamental Theory of Algebra.

Fischer: Intermediate Real Analysis.

Flanigan/Kazdan: Calculus Two: Linear and Nonlinear Functions. Second edition.

Fleming: Functions of Several Variables. Second edition.

Foulds: Combinatorial Optimization for Undergraduates.

Foulds: Optimization Techniques: An Introduction.

(continued after index) 
Johannes Buchmann

\section{Introduction to Cryptography}

Second Edition 
Johannes A. Buchmann

Department of Computer Science

Technical University, Darmstadt

Hochschulstr, 10

64289 Darmstadt

Germany

Editorial Board

S. Axler

Mathematics Department

San Francisco State

University

San Francisco, CA 94132

USA

axler@sfsu.edu

\author{
F.W. Gehring \\ Mathematics Department \\ East Hall \\ University of Michigan \\ Ann Arbor, MI 48109 \\ USA \\ gehring@math.lsa.umich.edu
}

K.A. Ribet

Mathematics Department

University of California

Berkeley

Berkeley, CA 94720-3840

USA

ribet@math.berkeley.edu

Cover: The factorization of RSA-576, a 576-bit or 174-digit prime number, was the goal of an open challenge sponsored by RSA Laboratories (Bedford, Mass.). RSA-576 was factored by a team of researchers in Germany and other countries in December, 2003.

Mathematics Subject Classification (2000): 94-01, 94A60, 11T71

Library of Congress Cataloging in Publication Data

Buchmann, Johannes.

Introduction to cryptography / Johannes Buchmann. - [2nd ed.].

p. cm. - (Undergraduate texts in mathematics)

Includes bibliographical references and index.

1. Coding theory. 2. Cryptography. I. Title. II Series.

QA268.B83 2004

$003^{\prime} .54-\mathrm{dc} 22 \quad 2004041657$

ISBN 978-0-387-20756-8 ISBN 978-1-4419-9003-7 (eBook)

DOI 10.1007/978-1-4419-9003-7

Printed on acid-free paper.

German edition: Einfürung in die Kryptographie ${ }^{\oplus}$ Springer Science+Business Media New York 2004

Originally published by Springer-Verlag New York, Inc. in 2004

Softcover reprint of the hardcover 1st edition

All rights reserved. This work may not be translated or copied in whole or in part without the written permission of the publisher (Springer Science+Business Media, LLC), except for brief excerpts in connection with reviews or scholarly analysis. Use in connection with any form of information storage and retrieval, electronic adaptation, computer software, or by similar or dissimilar methodology now known or hereafter developed is forbidden.

The use in this publication of trade names, trademarks, service marks, and similar terms, even if they are not identified as such, is not to be taken as an expression of opinion as to whether or not they are subject to proprietary rights.

98654321 SPIN 10991503 (hard cover) - SPIN 10963999 (soft cover)

springeronline.com 
For Almut, Daniel, and Jan 


\section{Contents}

Preface for the Second Edition

xiii

$\begin{array}{ll}\text { Preface } & \text { xv }\end{array}$

1 Integers 1

$1.1 \quad$ Basics . . . . . . . . . . . . . . . . 1

1.2 Divisibility . . . . . . . . . . . . . . 3

1.3 Representation of Integers . . . . . . . . . . . . 4

$1.4 \quad \mathrm{O}$ - and $\Omega$-Notation . . . . . . . . . . . . . . . 6

1.5 Cost of Addition, Multiplication, and Division with Remainder . . . . . . . . . . . . . 7

1.6 Polynomial Time . . . . . . . . . . . . . . . 9

1.7 Greatest Common Divisor . . . . . . . . . . . . . 9

1.8 Euclidean Algorithm . . . . . . . . . . . . . . . 12

1.9 Extended Euclidean Algorithm . . . . . . . . . . . 16

1.10 Analysis of the Extended Euclidean Algorithm . . . 18

1.11 Factoring into Primes . . . . . . . . . . . . 22

1.12 Exercises . . . . . . . . . . . . . . . 24

2 Congruences and Residue Class Rings 29

2.1 Congruences . . . . . . . . . . . . . . . . . 29 
2.2 Semigroups . . . . . . . . . . . . . . . . . 32

2.3 Groups ................... . . . 34

2.4 Residue Class Ring . . . . . . . . . . . . . . . . 35

2.5 Fields . . . . . . . . . . . . . . . . 36

2.6 Division in the Residue Class Ring . . . . . . . . . 36

2.7 Analysis of the Operations in the Residue Class Ring 38

2.8 Multiplicative Group of Residues $\bmod m \ldots . . . \quad 39$

2.9 Order of Group Elements . . . . . . . . . . . . . . 41

2.10 Subgroups . . . . . . . . . . . . . . . 42

2.11 Fermat's Little Theorem . . . . . . . . . . . . 44

2.12 Fast Exponentiation . . . . . . . . . . . . . 45

2.13 Fast Evaluation of Power Products . . . . . . . . . 48

2.14 Computation of Element Orders . . . . . . . . . 49

2.15 The Chinese Remainder Theorem . . . . . . . . . 51

2.16 Decomposition of the Residue Class Ring . . . . . . 53

2.17 A Formula for the Euler $\varphi$-Function . . . . . . . . 55

2.18 Polynomials . . . . . . . . . . . . . 56

2.19 Polynomials over Fields . . . . . . . . . . . . 58

2.20 Construction of Finite Fields . . . . . . . . . . 61

2.21 The Structure of the Unit Group of Finite Fields . . 65

2.22 Structure of the Multiplicative Group of Residues Modulo a Prime Number . . . . . . . . . . . . . 66

2.23 Exercises . . . . . . . . . . . . . . . . 67

3 Encryption $\quad \mathbf{7 1}$

3.1 Encryption Schemes . . . . . . . . . . . . . 71

3.2 Symmetric and Asymmetric Cryptosystems . . . . 73

3.3 Cryptanalysis . . . . . . . . . . . . . . . 74

3.4 Alphabets and Words . . . . . . . . . . . . . 77

3.5 Permutations . . . . . . . . . . . . . . . 80

3.6 Block Ciphers . . . . . . . . . . . . . . . . . . . 81

3.7 Multiple Encryption . . . . . . . . . . . . . . 82

3.8 The Use of Block Ciphers . . . . . . . . . . . . . . . . 83

3.9 Stream Ciphers . . . . . . . . . . . . . . . . . . . . 93

3.10 The Affine Cipher . . . . . . . . . . . . . . . . . 95

3.11 Matrices and Linear Maps . . . . . . . . . . . . . 97

3.12 Affine Linear Block Ciphers . . . . . . . . . . . 102

3.13 Vigenère, Hill, and Permutation Ciphers . . . . . . 103 
3.14 Cryptanalysis of Affine Linear Block Ciphers . . . . 104

3.15 Secure Cryptosystems . . . . . . . . . . . . . . 105

3.16 Exercises . . . . . . . . . . . . . . . . . 111

4 Probability and Perfect Secrecy 115

4.1 Probability . . . . . . . . . . . . . . . . . 115

4.2 Conditional Probability . . . . . . . . . . . 117

4.3 Birthday Paradox . . . . . . . . . . . . . . . . 118

4.4 Perfect Secrecy . . . . . . . . . . . . . . . . . . . . . . 119

4.5 Vernam One-Time Pad . . . . . . . . . . . . . 123

4.6 Random Numbers . . . . . . . . . . . . . . . . . . . 124

4.7 Pseudorandom Numbers . . . . . . . . . . . . . . . 124

4.8 Exercises . . . . . . . . . . . . . . . . . 125

5 DES 127

5.1 Feistel Ciphers . . . . . . . . . . . . . . . . 127

5.2 DES Algorithm . . . . . . . . . . . . . . . . . . 128

5.3 An Example . . . . . . . . . . . . . . . . . . . . 134

5.4 Security of DES . . . . . . . . . . . . 136

5.5 Exercises . . . . . . . . . . . . . . . 137

6 AES 139

6.1 Notation . . . . . . . . . . . . . . . . . . 139

6.2 Cipher . . . . . . . . . . . . . . . 140

6.3 KeyExpansion . . . . . . . . . . . . . . . 145

6.4 An Example . . . . . . . . . . . . . . . . . . . . . 146

6.5 InvCipher . . . . . . . . . . . . . . 148

6.6 Exercises . . . . . . . . . . . . . . . . 148

7 Prime Number Generation 151

7.1 Trial Division . . . . . . . . . . . . . . . . 151

$7.2 \quad$ Fermat Test . . . . . . . . . . . . . . . . 153

7.3 Carmichael Numbers . . . . . . . . . . . . . . . 154

7.4 Miller-Rabin Test . . . . . . . . . . . . . . . 156

7.5 Random Primes . . . . . . . . . . . . . . . . . 159

7.6 Exercises . . . . . . . . . . . . . . . 160 
8 Public-Key Encryption $\quad 163$

8.1 Idea . . . . . . . . . . . . . . . . . 163

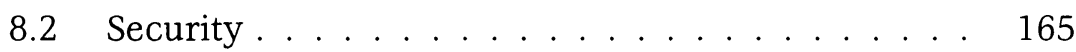

8.3 RSA Cryptosystem . . . . . . . . . . . . . . . . . 167

8.4 Rabin Encryption . . . . . . . . . . . . . . . . 181

8.5 Diffie-Hellman Key Exchange . . . . . . . . . . . 186

8.6 ElGamal Encryption . . . . . . . . . . . . . . . . . . 191

8.7 Exercises . . . . . . . . . . . . . . . . 196

9 Factoring 199

9.1 Trial Division . . . . . . . . . . . . . . . . . . 199

$9.2 p-1$ Method . . . . . . . . . . . . . . . 200

9.3 Quadratic sieve ................. 201

9.4 Analysis of the Quadratic Sieve . . . . . . . . . 206

9.5 Efficiency of Other Factoring Algorithms . . . . . . 210

9.6 Exercises . . . . . . . . . . . . . . . 211

10 Discrete Logarithms 213

10.1 The DL Problem . . . . . . . . . . . . . . . . . 213

10.2 Enumeration . . . . . . . . . . . . . . . . 214

10.3 Shanks Baby-Step Giant-Step Algorithm . . . . . . . 214

10.4 The Pollard $\rho$-Algorithm . . . . . . . . . . . 217

10.5 The Pohlig-Hellman Algorithm . . . . . . . . . . 221

10.6 Index Calculus . . . . . . . . . . . . . . . 226

10.7 Other Algorithms . . . . . . . . . . . . . . 230

10.8 Generalization of the Index Calculus Algorithm . . 231

10.9 Exercises . . . . . . . . . . . . . . . 232

11 Cryptographic Hash Functions 235

11.1 Hash Functions and Compression Functions . . . . 235

11.2 Birthday Attack . . . . . . . . . . . . . . . 238

11.3 Compression Functions from Encryption Functions 239

11.4 Hash Functions from Compression Functions . . . 239

11.5 SHA-1 . . . . . . . . . . . . . . . 242

11.6 Other Hash Functions . . . . . . . . . . . . . . . . . 244

11.7 An Arithmetic Compression Function . . . . . . . 245

11.8 Message Authentication Codes . . . . . . . . . . . 247

11.9 Exercises . . . . . . . . . . . . . . . . . . 248 
12 Digital Signatures $\quad 249$

12.1 Idea . . . . . . . . . . . . . . . . . . 249

12.2 Security . . . . . . . . . . . . . . 250

12.3 RSA Signatures . . . . . . . . . . . . . . . . . . 251

12.4 Signatures from Public-Key Systems . . . . . . . . 257

12.5 ElGamal Signature . . . . . . . . . . . . . . . . . 257

12.6 The Digital Signature Algorithm (DSA) . . . . . . 263

12.7 Undeniable Signatures . . . . . . . . . . . . . 266

12.8 Blind Signatures . . . . . . . . . . . . . . . . . . 271

12.9 Exercises . . . . . . . . . . . . . . . 274

13 Other Systems 277

13.1 Finite Fields . . . . . . . . . . . . . . . . . . . . 278

13.2 Elliptic Curves . . . . . . . . . . . . . . . . . . . 278

13.3 Quadratic Forms . . . . . . . . . . . . . . . . . 282

13.4 Exercises . . . . . . . . . . . . . . . . . . 283

14 Identification $\quad 285$

14.1 Passwords . . . . . . . . . . . . . . . . 286

14.2 One-Time Passwords . . . . . . . . . . . . . . 287

14.3 Challenge-Response Identification . . . . . . . . . 287

14.4 Exercises . . . . . . . . . . . . . . . . . . 292

15 Secret Sharing 293

15.1 The Principle . . . . . . . . . . . . . . . . . . 293

15.2 The Shamir Secret Sharing Protocol . . . . . . . . 294

15.3 Exercises . . . . . . . . . . . . . . . . . . 297

16 Public-Key Infrastructures 299

16.1 Personal Security Environments . . . . . . . . . . 299

16.2 Certification Authorities . . . . . . . . . . . . . 301

16.3 Certificate Chains . . . . . . . . . . . . . . 306

$\begin{array}{ll}\text { Solutions of the exercises } & 307\end{array}$

$\begin{array}{ll}\text { References } & 325\end{array}$

$\begin{array}{ll}\text { Index } & 331\end{array}$ 


\section{Preface for the Second Edition}

The second edition of my introduction to cryptography contains updates and new material. I have updated the discussion of the security of encryption and signature schemes and the state of the art in factoring and computing discrete logarithms. I have added descriptions of time-memory trade of attacks and algebraic attacks on block ciphers, the Advanced Encryption Standard (AES), the Secure Hash Algorithm (SHA-1), secret sharing schemes, and undeniable and blind signatures. I have also corrected the errors that have been reported to me. I thank the readers of the first edition for all comments and suggestions.

October 2003

Johannes Buchmann 


\section{Preface}

Cryptography is a key technology in electronic security systems. Modern cryptograpic techniques have many uses, such as to digitally sign documents, for access control, to implement electronic money, and for copyright protection. Because of these important uses it is necessary that users be able to estimate the efficiency and security of cryptographic techniques. It is not sufficient for them to know only how the techniques work.

This book is written for readers who want to learn about modern cryptographic algorithms and their mathematical foundation but who do not have the necessary mathematical background. It is my goal to explain the basic techniques of modern cryptography, including the necessary mathematical results from linear algebra, algebra, number theory, and probability theory. I only assume basic mathematical knowledge.

The book is based on courses in cryptography that I have been teaching at the Technical University Darmstadt, since 1996. I thank all students who attended the courses and who read the manuscript carefully for their interest and support. In particular, I would like to thank Harald Baier, Gabi Barking, Manuel Breuning, Safuat Hamdy, Birgit Henhapl, Michael Jacobson (who also corrected my English), Markus Maurer, Andreas Meyer, Stefan Neis, Sachar Paulus, Thomas 
Pfahler, Marita Skrobic, Edlyn Teske, Patrick Theobald, and RalfPhilipp Weinmann. I also thank the staff at Springer-Verlag, in particular Martin Peters, Agnes Herrmann, Claudia Kehl, Ina Lindemann, and Terry Kornak, for their support in the preparation of this book.

Darmstadt

June 1999

Johannes Buchmann 